\title{
Thyroid-stimulating hormone levels in euthyroid patients 8 years following bariatric surgery
}

\author{
Anne Lautenbach (iD ${ }^{1 凶}$, Marie Wernecke ${ }^{1}$, Oliver Mann ${ }^{2}$, Philipp Busch ${ }^{2}$, Tobias B. Huber ${ }^{1}$, Fabian Stoll (iD ${ }^{1,3}$ and Jens Aberle ${ }^{1,3}$
}

(c) The Author(s) 2022

BACKGROUND: Bariatric surgery (BS) was shown to promote a decline in thyroid-stimulating hormone (TSH) in euthyroid patients with severe obesity in the short-term. Aim of the present study was to assess the effect of weight loss on thyroid function in euthyroid patients in the long-term following different bariatric procedures.

METHODS: In a retrospective cohort study including 135 patients at baseline, thyroid function was assessed at six time points up to 8 years after surgery. Patients were stratified by TSH levels at baseline and divided into two groups to compare the change in TSH at long-time. We used log-linear regression to assess the relation between thyroid hormones and TSH and linear regression analyses to identify variables that were thought to determine TSH and fT3/fT4-ratio as well as their change long-term.

RESULTS: Over a mean follow-up of 8 years, TSH and fT3/fT4-ratio declined (both $p<0.001$ ). Patients with high-normal TSH showed a greater decline in TSH than those with normal TSH compared to baseline. Thyroid hormones and TSH displayed a negative loglinear correlation at long-term follow-up. Change in TSH at long-time showed a negative correlation with TSH at baseline $(B=$ $-0.55 ; p<0.001)$. With regard to type of surgery, there were no significant differences in TSH.

CONCLUSION: BS promotes a decline of TSH in euthyroid patients up to 8 years after intervention despite weight regain. The greatest change in TSH was seen among patients with high-normal baseline-TSH. Results of log-linear regression suggest recovery of the pituitary-thyroid axis. Type of surgery did not affect the change in TSH levels over time.

International Journal of Obesity (2022) 46:825-830; https://doi.org/10.1038/s41366-021-01058-z

\section{INTRODUCTION}

In the last few decades the prevalence of obesity constantly increased reaching pandemic levels. The WHO reported a prevalence of $13 \%$ for adults with obesity in 2016 [1]. Besides commonly used lifestyle interventions bariatric surgery (BS) has become an effective treatment option for patients with severe obesity [2].

Severe obesity has previously shown to be associated with high-normal serum levels of thyroid stimulating hormone (TSH) and free triiodothyronine (fT3) in euthyroid adults [3]. Subtle changes in thyroid hormones, even within the normal range, adversely influence cardiovascular, bone and metabolic outcomes $[4,5]$. High-normal TSH-values are associated with an adverse lipid profile, high blood pressure, high body mass index (BMI), metabolic syndrome and fatal coronary heart disease [4]. However, the causal relationship between severe obesity and thyroid function still remains unclear $[6,7]$. Patients with severe obesity may have transient hypothyroidism caused by an increase in leptin-levels that resolves following Roux-en- $Y$ gastric bypass (RYGB) [8].

There are only few studies addressing the relation between change in TSH levels and change in body weight in the euthyroid range following BS [9-14], even though the majority of bariatric patients have normal thyroid function [8]. Short-term studies have shown that weight loss induced by BS promotes a significant decline of increased TSH levels in euthyroid patients with severe obesity [9-11]. This initial decline was progressive over time and associated with excess BMI loss [9]. However, in most studies, thyroid parameters were analyzed after up to 12 months postsurgery $[9-13,15]$. Only three studies had a follow-up of 24 months $[14,16,17]$. To the best of our knowledge, there are no studies addressing variations in TSH within the euthyroid range following BS in the long-term. Moreover, no data exist investigating the impact of surgery technique on thyroid metabolism in the longterm.

Therefore, the primary aim of the study was to investigate the effect of weight loss on TSH levels in euthyroid patients in the long-term following different bariatric procedures.

\section{MATERIALS AND METHODS \\ Study population}

Euthyroid male or female patients $\geq 18$ years who underwent either sleeve gastrectomy (SG) or RYGB according to the S3 Guidelines Chirurgie der Adipositas [18] were included in the analysis. Patients with second-step procedures were considered as having SG $(n=2)$. Patients attended our obesity outpatient clinic between 2008-2020, which is certified as center of excellence for obesity and metabolic surgery by the European Accreditation Council for Bariatric Surgery. Exclusion criteria included incomplete records, history of autoimmune thyroid disease, history of acute inflammation (pulmonary, gastrointestinal, urogenital, or cutaneous),

\footnotetext{
${ }^{1}$ III Department of Medicine, University Medical Center Hamburg-Eppendorf, Hamburg, Germany. ${ }^{2}$ Department of General, Visceral and Thoracic Surgery, University Medical
} Center Hamburg-Eppendorf, Hamburg, Germany. ${ }^{3}$ These authors contributed equally: Fabian Stoll, Jens Aberle. ${ }^{凶}$ email: a.lautenbach@uke.de 
history of chronic autoinflammatory disease, pituitary insufficiency, pregnancy and treatment with thyreostatic drugs, thyroid hormones, amiodarone or lithium. Blood samples were taken in the afternoon in a non-fasting condition. Of an initial population of 330 patients, 195 were excluded $(n=135)$.

All procedures performed in this study involving human participants were in accordance with the ethical standards of the institutional and/or national committee on human research and with the 1964 World Medical Association Declaration of Helsinki and its later amendments or comparable ethical standards. Retrospective data collection and anonymized analysis was conducted in accordance with local government law (HmbKHG. §12) without the requirement for informed consent.

\section{Study design}

Follow-up data were retrospectively collected from 135 patients at baseline. To provide reasonable comparability between the cases, the available data were allocated 5 "visits" by time in relation to the procedure. In addition to baseline data $8.07 \pm 5.26$ (mean \pm SD) months before surgery $(n=135)$, data from visit $1(n=132)$ were analyzed 6 months after surgery, data from visit $2(n=110) 1$ year after surgery, data from visit $3(n=97) 2$ years after surgery, data from visit $4(n=72) 3$ years after surgery and data from visit $5(n=52) 8$ years post BS. Over the follow-up period 82 patients were lost to follow-up.

\section{Variables}

Data on height, weight, BMl, sex, age, and serum levels of TSH, fT3, free thyroxine (fT4), glycated hemoglobin ( $\mathrm{HbA} 1 \mathrm{c})$, total cholesterol, triglycerides, low-density lipoprotein cholesterol (LDL-cholesterol), high-density lipoprotein cholesterol (HDL-cholesterol), C-reactive protein (CRP), aspartate aminotransferase (AST), alanine aminotransferase (ALT), and gammaglutamyl transferase (GGT) were analyzed at baseline and during follow-up. Data on cardiovascular comorbidities were recorded at baseline and data on post-surgical complications were recorded over the follow-up period. fT3 and fT4 were measured by a sandwich chemiluminescence homogeneous immunoassay (LOCl-technology, Dimension Vista 1500 Analyzer, Siemens Healthineers). The normal range for serum fT3 levels and fT4 levels was $3.5-6.5 \mathrm{pmol} / \mathrm{l}$ and $11.5-22.7 \mathrm{pmol} / \mathrm{l}$, respectively. TSH was measured by a sequenced chemiluminescence homogeneous immunoassay (LOCI-technology, Dimension Vista 1500 Analyzer, Siemens Healthineers). The normal range for serum TSH levels was $0.55-4.78 \mathrm{mU} / \mathrm{l}$. The fT3/ fT4-ratio as an indirect index of D1 and D2 deiodinase activity was calculated [16]. Excess weight loss (EWL) in \% was calculated by dividing the difference between initial and final BMI by the difference between initial BMI and a target BMI of $25 \mathrm{~kg} / \mathrm{m}^{2}$. Optimal weight loss was defined as losing at least $50 \%$ of the excess weight during the first 2 years after the procedure $[19,20]$. We computed the change in TSH levels and fT3/fT4ratio as the change between baseline and each follow-up visit.

\section{Subgroup classification}

Patients were stratified by TSH-levels at baseline and divided into two groups based on previous studies that indicate that more than $95 \%$ of healthy individuals have TSH levels below $2.5 \mathrm{mU} / \mathrm{I}[21,22]$ : (1) normal TSH $(<2.5 \mathrm{mU} / \mathrm{l})$ and (2) high-normal TSH $(\geq 2.5 \mathrm{mU} / \mathrm{l})$. We also performed subgroup analyses to compare the change in thyroid function between different surgical approaches (SG vs. RYGB) as well as the presence and absence of treatment requiring hypertension.

\section{Statistical analysis}

Independent continuous variables were analyzed fitting a mixed-effects model for repeated measures treating missing values of lost to follow-up patients as random effect. The model uses a compound symmetry covariance matrix and is fit using restricted maximum likelihood. Tukey's test was used to correct for multiple comparisons. Non-sphericity of variables was corrected using the Geisser-Greenhouse method. Subgroups were added as additional factor to the model using Fisher's least significant difference test for comparisons between subgroups.

Log-linear regression between log-transformed TSH-levels and thyroid hormones was performed for each visit. Linear regression and multiple linear regression analysis were performed to identify independent variables that determined TSH-levels and fT3/fT4-ratios as well as their changes compared to baseline for all visits and compared to visit 4 at longterm.

Continuous variables are expressed as mean \pm SD. Categorical variables are presented as absolute or relative numbers. For all statistical tests a $p<$ $0.05\left({ }^{*} p<0.05,{ }^{* *} p<0.01\right.$ and $\left.{ }^{* * *} p<0.001\right)$ was considered statistically significant. A minimal sample size of 53 was calculated assuming an effect size of Cohen's $f=0.25$ with a type I error of $a=0.05$, a statistical power of 0.95 , number of repeated measurements of 6 , a correlation among repeated measures of 0.5 and an assumed correction for non-sphericity of $\varepsilon=0.4$ with $G *$ Power, version 3.1.9.7 for Windows [23]. All analysis and graphical representation were performed via GraphPad Prism 9, version 9.2.0 for Windows, GraphPad Software Inc., California, United States.

\section{RESULTS}

\section{Demographic and biochemical data follow-up at baseline}

Mean age at baseline was $42.1 \pm 10.6$ years, $68.9 \%$ of patients were female. In total, $45.2 \%$ of patients underwent SG, 54.8\% RYGB as initial weight loss procedure. Demographic data for baseline and follow-up visits is presented in Table 1. At baseline, $49.8 \%$ of patients suffered from treatment requiring hypertension, $2.2 \%$ from coronary heart disease, and 3.0\% from cardiac arrythmia.

Post-surgical complications included the development of dumping syndrome (5.9\% of patients) as well as gastroesophageal reflux disease (1.5\% of patients).

At baseline, mean BMI was $50.8 \pm 8.6 \mathrm{~kg} / \mathrm{m}^{2}$ and $\mathrm{HbA} 1 \mathrm{c}$ was $6.3 \pm 1.2 \%$. At baseline, TSH was $2.06 \pm 0.79 \mathrm{mU} / \mathrm{l}$, fT3/fT4-ratio was $0.33 \pm 0.05$. Biochemical data for baseline and follow-up visits is presented in Table 2.

Comparing SG and RYGB, TSH was not significantly different between the groups at baseline $(2.06 \pm 0.84$ vs. $2.07 \pm 0.75 \mathrm{mU} / \mathrm{l}$, $p=0.90)$.

Comparing between the presence and absence of treatment requiring hypertension at baseline, there was a slight difference by trend in TSH between the groups at baseline, although not statistically significant $(1.96 \pm 0.69$ vs. $2.16 \pm 0.87, p=0.098)$.

When evaluating by baseline-TSH, the groups did not differ significantly in any other parameter than TSH. Normal TSH-levels with a mean of $1.63 \pm 0.45 \mathrm{mU} / \mathrm{l}$ was present in $68.9 \%$ of patients. Patients were $41.9 \pm 12.1$ years, BMl was $50.7 \pm 8.7 \mathrm{~kg} / \mathrm{m}^{2}$, $\mathrm{HbA} 1 \mathrm{c}$ was $6.3 \pm 1.2 \%$. High-normal TSH-levels with a mean of $3.02 \pm$

Table 1. Demographic characteristics of study population at baseline and follow-up visits.

\begin{tabular}{|c|c|c|c|c|c|c|}
\hline & Baseline & Visit 1 & Visit 2 & Visit 3 & Visit 4 & Visit 5 \\
\hline$N$ & 135 & 132 & 110 & 97 & 72 & 52 \\
\hline $\operatorname{Sex}(F, n)$ & 93 & 92 & 76 & 71 & 55 & 39 \\
\hline Age (years) & $42.1 \pm 10.6$ & $43.3 \pm 10.4$ & $43.8 \pm 10.5$ & $45.6 \pm 9.3$ & $46.1 \pm 9.8$ & $52.6 \pm 8.9$ \\
\hline SG $(n)$ & 61 & 58 & 51 & 44 & 33 & 22 \\
\hline RYGB $(n)$ & 74 & 74 & 59 & 53 & 39 & 30 \\
\hline Time to BS (months) & $-8.07 \pm 5.26$ & $6.06 \pm 1.19$ & $11.90 \pm 1.32$ & $23.32 \pm 2.60$ & $35.80 \pm 2.57$ & $95.60 \pm 6.97$ \\
\hline Optimal weight loss (\%) & 71.9 & 72.0 & 80.3 & 72.2 & 75.0 & 76.9 \\
\hline
\end{tabular}

Data are reported as mean $\pm S D$.

$N$ number of individuals, $F$ female, SG sleeve gastrectomy, RYGB Roux-en-Y gastric bypass, $B S$ bariatric surgery. 
Table 2. Anthropometric and biochemical characteristics at baseline and follow-up visits.

\begin{tabular}{|c|c|c|c|c|c|c|}
\hline & Baseline & Visit 1 & Visit 2 & Visit 3 & Visit 4 & Visit 5 \\
\hline Weight (kg) & $150.8 \pm 34.2$ & $115.3 \pm 27.7^{* * *}$ & $109.6 \pm 30.0^{* * *}$ & $105.4 \pm 26.1^{* * *}$ & $103.4 \pm 26.4^{* * *}$ & $114.6 \pm 30.8^{* * *}$ \\
\hline BMI $\left(\mathrm{kg} / \mathrm{m}^{2}\right)$ & $50.8 \pm 8.6$ & $38.9 \pm 7.3^{* * *}$ & $36.9 \pm 8.2^{* * *}$ & $35.8 \pm 7.5^{* * *}$ & $35.3 \pm 7.8^{* * *}$ & $38.6 \pm 8.5^{* * *}$ \\
\hline EWL (\%) & & $48.9 \pm 16.4$ & $58.7 \pm 22.4$ & $60.3 \pm 23.6$ & $63.1 \pm 23.0$ & $46.1 \pm 34.7$ \\
\hline $\mathrm{TSH}(\mathrm{mU} / \mathrm{l})$ & $2.06 \pm 0.79$ & $1.61 \pm 0.78^{* * *}$ & $1.55 \pm 0.70^{* * *}$ & $1.32 \pm 0.62^{* * *}$ & $1.34 \pm 0.56^{* * *}$ & $1.35 \pm 0.62^{* * *}$ \\
\hline fT3 (pmol/l) & $5.0 \pm 0.6$ & $4.5 \pm 0.6^{* * *}$ & $4.4 \pm 0.6^{* * *}$ & $4.4 \pm 0.6^{* * *}$ & $4.3 \pm 0.5^{* * *}$ & $4.0 \pm 0.6^{* * *}$ \\
\hline fT4 (pmol/l) & $15.4 \pm 2.4$ & $16.2 \pm 2.7^{* *}$ & $16.0 \pm 2.1^{*}$ & $16.2 \pm 1.9^{*}$ & $16.5 \pm 2.1^{* * *}$ & $15.9 \pm 2.3$ \\
\hline fT3/fT4 & $0.33 \pm 0.05$ & $0.28 \pm 0.05^{* * *}$ & $0.28 \pm 0.04^{* * *}$ & $0.28 \pm 0.04^{* * *}$ & $0.26 \pm 0.04^{* * *}$ & $0.25 \pm 0.04^{* * *}$ \\
\hline HbA1c (\%) & $6.3 \pm 1.2$ & $5.6 \pm 0.7^{* * *}$ & $5.6 \pm 0.7^{* * *}$ & $5.8 \pm 1.0^{* * *}$ & $5.6 \pm 1.1^{* * *}$ & $5.9 \pm 1.1^{* *}$ \\
\hline Total cholesterol (mg/dl) & $207 \pm 35$ & $189 \pm 36^{* * *}$ & $188 \pm 33^{* * *}$ & $189 \pm 33^{* * *}$ & $186 \pm 33^{* * *}$ & $191 \pm 32^{* * *}$ \\
\hline Triglycerides (mg/dl) & $203 \pm 123$ & $138 \pm 82^{* * *}$ & $140 \pm 70^{* * *}$ & $145 \pm 72^{* * *}$ & $128 \pm 70^{* * * *}$ & $141 \pm 73^{* * *}$ \\
\hline LDL-cholesterol (mg/dl) & $119 \pm 30$ & $112 \pm 33^{* *}$ & $101 \pm 27^{* * *}$ & $100 \pm 32^{* * *}$ & $98 \pm 29^{* * *}$ & $99 \pm 30^{* * *}$ \\
\hline HDL-cholesterol (mg/dl) & $51 \pm 15$ & $51 \pm 14$ & $57 \pm 16^{* * *}$ & $61 \pm 18^{* * *}$ & $64 \pm 21^{* * *}$ & $62 \pm 16^{* * *}$ \\
\hline CRP (mg/l) & $10.2 \pm 7.4$ & $7.0 \pm 4.6^{* * *}$ & $6.1 \pm 2.8^{* * *}$ & $5.9 \pm 2.8^{* * * *}$ & $6.3 \pm 3.7^{* * * *}$ & $5.4 \pm 2.2^{* * *}$ \\
\hline AST (U/I) & $33 \pm 20$ & $23 \pm 9^{* * *}$ & $20 \pm 8^{* * *}$ & $19 \pm 7^{* * *}$ & $20 \pm 10^{* * *}$ & $20 \pm 6^{* * *}$ \\
\hline ALT (U/I) & $38 \pm 25$ & $24 \pm 13^{* * *}$ & $20 \pm 10^{* * *}$ & $20 \pm 8^{* * *}$ & $21 \pm 11^{* * *}$ & $26 \pm 9 * * *$ \\
\hline GGT (U/I) & $44 \pm 33$ & $27 \pm 20^{* * *}$ & $25 \pm 16^{* * *}$ & $26 \pm 19^{* * *}$ & $27 \pm 22^{* * *}$ & $24 \pm 12^{* * *}$ \\
\hline
\end{tabular}

Data are reported as mean \pm SD. A mixed-effects model with Tukey's multiple comparisons test was used to compare follow-up visits with baseline for continuous variables $\left({ }^{*} p<0.05,{ }^{* *} p<0.01\right.$ and $\left.{ }^{* * *} p<0.001\right)$.

$B M I$ body mass index, EWL excess weight loss, $T S H$ thyroid stimulating hormone, $f T 3$ free triiodothyronine, fT4 free thyroxine, $H b A 1 c$ glycated hemoglobin, $L D L$ low-density lipoprotein, HDL high-density lipoprotein, CRP C-reactive protein, AST aspartate aminotransferase, ALT alanine aminotransferase, GGT gammaglutamyl transferase.

$0.47 \mathrm{mU} / \mathrm{l}$ was present in $31.1 \%$ of patients. Patients were $42.2 \pm$ 9.87 years, BMI was $51.0 \pm 8.4 \mathrm{~kg} / \mathrm{m}^{2}, \mathrm{HbA} 1 \mathrm{c}$ was $6.2 \pm 1.2 \%$.

\section{Weight loss outcomes 8 years after surgery}

Body weight and BMl declined during follow-up compared to baseline ( $p<0.001$, all visits). Weight regain at visit 5 was statistically significant compared to visit $4(p<0.001)$ (Table 2$)$. The mean EWL at long-term was $46.1 \pm 34.7 \%$. At visit $5,76.9 \%$ of patients had achieved optimal weight loss of greater than $50 \%$ EWL during the first 2 years after surgery (Table 1).

Comparing SG and RYGB, EWL was not significantly different between the groups at long-term $(43.3 \pm 13.7$ vs. $48.1 \pm 44.4 \%$, $p=0.58)$.

There was no significant difference between patients with normal and high-normal TSH baseline-levels in body weight $(116.4 \pm 31.3$ vs. $110.9 \pm 30.3 \mathrm{~kg}, p=0.55)$, BMI $(39.3 \pm 8.6$ vs. $\left.37.2 \pm 8.5 \mathrm{~kg} / \mathrm{m}^{2}, p=0.41\right)$ and EWL (41.2 \pm 39.5 vs. $56.2 \pm 19.2 \%$, $p=0.07)$ at visit 5

\section{Glycemic control 8 years after surgery}

$\mathrm{HbA1C}$ significantly decreased over the whole follow-up period compared to baseline $(p<0.001$, visit $1-4 ; p=0.005$, visit 5$)$. A gradual re-increase occurred at long-term, which was not statistically significant compared to visit $4(p=0.054)$ (Table 2$)$.

Comparing SG and RYGB, HbA1c was not significantly different between the groups at long-term (5.7 \pm 0.8 vs. $6.0 \pm$ $1.2 \%, p=0.26$ ).

When evaluating by baseline-TSH, HbA1c did not differ significantly between patients with normal and high-normal baseline-levels at visit 5 ( $5.6 \pm 0.8$ vs. $6.0 \pm 1.1 \%, p=0.16)$.

Metabolic and cardiovascular parameters 8 years after surgery Total cholesterol and triglycerides decreased over the whole follow-up-period compared to baseline ( $p<0.001$, all visits) (Table 2 ). A gradual re-increase at long-term was not statistically significant compared to visit 4 for both parameters ( $p=0.214$ and $p=0.053$ ). LDL-cholesterol statistically decreased during follow-up compared to baseline $(p=0.007$, visit $1 ; p<0.001$, visit $2-5)$, while HDLcholesterol remained stable at visit 1 compared to baseline ( $p=$ 0.498 ) and statistically increased over the remaining follow-up period compared to baseline $(p<0.001$, visit $2-5)$ (Table 2$)$.

Mean serum levels of CRP statistically declined over the whole follow-up period compared to baseline ( $p<0.001$, all visits) (Table 2$)$ and showed a statistically significant re-increase at long-term compared to visit 4 ( $p=0.013)$.

Serum levels of AST, ALT, and GGT dropped over the whole follow-up period compared to baseline $(p<0.001$, all visits) (Table 2). ALT showed a statistically significant re-increase at long-term compared to visit $4(p=0.001)$, while AST and GGT remained stable at long-term compared to visit $4(p=0.944$ and $p=0.107)$.

\section{Thyroid function outcomes $\mathbf{8}$ years after surgery}

TSH-levels and fT3/fT4-ratio declined over the follow-up period compared to baseline ( $p<0.001$, all visits). At visit 5, TSH remained stable compared to visit 4 (1.34 \pm 0.56 vs. $1.35 \pm 0.62 \mathrm{mU} / \mathrm{l}, p=$ $0.80)$ and fT3/fT4-ratio remained stable compared to visit $4(0.26 \pm$ 0.04 vs. $0.25 \pm 0.04, p=0.57$ ) (Table 2 ).

Comparing SG and RYGB, there were no significant differences in TSH (1.25 \pm 0.60 vs. $1.43 \pm 0.63 \mathrm{mU} / \mathrm{l}, p=0.29)$ and fT3/fT4-ratio $(0.26 \pm 0.04$ vs. $0.25 \pm 0.03, p=0.33)$ between the surgical procedures at visit 5 .

Comparing between the presence and absence of treatment requiring hypertension at baseline, TSH was not statistically significant different between the groups at visit $5(1.21 \pm 0.59$ vs. $1.50 \pm 0.59, p=0.152$ ), while $\mathrm{fT} 3 / \mathrm{fT} 4$-ratio showed a statistically significant difference at visit $5(0.24 \pm 0.04$ vs. $0.27 \pm 0.03$, $p=0.022$ ).

When evaluating by baseline-TSH, TSH-levels as well as the corresponding $\mathrm{fT} 3 / \mathrm{fT} 4$-ratios decreased in patients with normal and high-normal TSH baseline-levels over time $(p<0.001$, all visits). The most pronounced decline in TSH-levels compared to baseline appeared at visit 1 for both groups (1.63 \pm 0.45 vs. $1.30 \pm$ $0.57, p<0.001$ and $3.02 \pm 0.47$ vs. $2.31 \pm 0.75, p<0.001)$ and 
remained stable at long-term follow-up (shown in Fig. 1). Patients with high-normal TSH-levels at baseline showed a greater change in TSH compared to baseline at long-time follow-up than patients with normal TSH-levels at baseline $(-1.16 \pm 0.70$ vs. $-0.39 \pm 0.51$, $p=0.002$ ). There was no significant difference in change of fT3/ fT4-ratio compared to baseline at visit 5 between the two groups $(-0.09 \pm 0.04$ vs. $-0.08 \pm 0.05, p=0.54)$.

\section{Log-linear regression analysis between TSH and thyroid hormones at long-time follow-up}

Log-linear regression analysis at long-time follow-up showed a negative log-linear correlation between fT3-levels and logtransformed TSH levels $\left(\log \mathrm{TSH}=3.78-4.09 \mathrm{fT} 3, r^{2}=0.19, p=\right.$ 0.001 ) (shown in Fig. 2a). Likewise, fT4-levels showed a negative correlation with TSH-levels at long-term-follow-up (log TSH = 5.13-3.16 fT4, $r^{2}=0.11, p=0.017$ ) (shown in Fig. 2b). Similar loglinear regression analysis at baseline and earlier follow-up visits showed no significant negative log-linear correlation between thyroid hormones and log-transformed TSH-levels, except at 1-year follow-up ( $\log \mathrm{TSH}=3.81-3.52 \mathrm{fT} 3, r^{2}=0.09, p=0.001$ and $\left.\log \mathrm{TSH}=5.10-2.96 \mathrm{fT} 4, r^{2}=0.05, p=0.013\right)$.

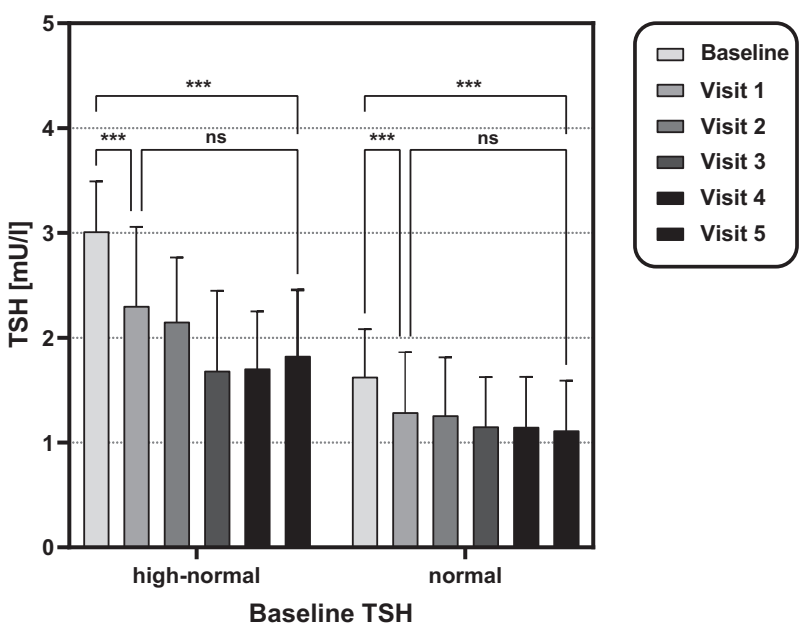

Fig. 1 Subgroup classification according to TSH-levels at baseline and postoperative course of TSH for both subgroups. TSH thyroid stimulating hormone. Results are expressed as means and standard deviation. ${ }^{*} p<0.05,{ }^{* *} p<0.01$ and ${ }^{* * *} p<0.001$.

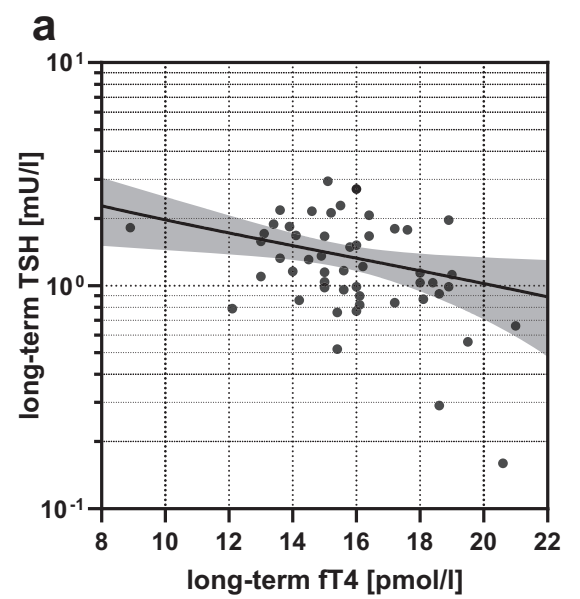

Linear regression analysis for change in TSH and for change in fT3/fT4-ratio at long-time follow-up

Change in TSH and change in fT3/fT4-ratio at long-time follow-up showed a negative correlation with TSH at baseline (shown in Fig. 3a) and fT3/fT4-ratio at baseline (shown in Fig. 3b), respectively $(B=-0.55, p<0.001$ and $B=-0.70, \quad p<0.001$; respectively). There was no statistically significant correlation between weight regain at long-term compared to visit 4 with change in TSH and change in fT3/fT4-ratio at long-time follow-up compared to visit $4(B=0.005, p=0.622$ and $B=0.029, p=0.270)$.

Multiple linear regression analysis for change in TSH and for change in fT3/fT4-ratio at long-time follow-up

Multiple linear regression showed that TSH at baseline was the only variable significantly associated with the change in TSH at visit 5. Change in TSH at long-time showed a negative correlation with TSH at baseline $(B=-0.47, p<0.001)$. Accordingly, fT3/fT4ratio at baseline was the only variable associated with the change in fT3/fT4-ratio at visit 5. Change in fT3/fT4-ratio at long-time follow-up showed a negative correlation with fT3/fT4-ratio at baseline $(B=-0.61, p<0.001)$.

\section{DISCUSSION}

This retrospective study is the first study providing long-term data about thyroid function in euthyroid patients with obesity after BS. We report a significant decline in TSH-levels and fT3/fTt4-ratio 8 years after surgery. Our data add knowledge about velocity, temporal dynamics and interrelationship to available short-term studies, in which a decline in TSH-levels 6-24 months after weight loss surgery has been observed [10, 11, 24, 25].

In the present cohort study, TSH-levels declined by $0.71 \mathrm{mU} / \mathrm{l}$ over a mean follow-up of 8 years. The most pronounced decline in TSH was observed during the first 6 months following BS. Observational studies showed that there is a positive association between $\mathrm{BMI}$ and $\mathrm{TSH}$, even within the normal range [26, 27]. Causality was assessed by a recent Mendelian randomization analysis, showing that thyroid function correlated to weight increases over 5 years. Thyroid function accounted for $1 \%$ of variation in BMI between individuals, having the same impact on BMI as physical activity [28]. In a cross-sectional population study, a difference in BMI of $1.9 \mathrm{~kg} / \mathrm{m}^{2}(5.5 \mathrm{~kg})$ between patients with the highest and lowest TSH-levels, but no overt thyroid dysfunction, was observed [26].

Moreover, change in TSH at long-time showed a strong negative correlation with TSH at baseline, which is in line with data 6-12 months after SG in euthyroid patients [10]. Change in

b

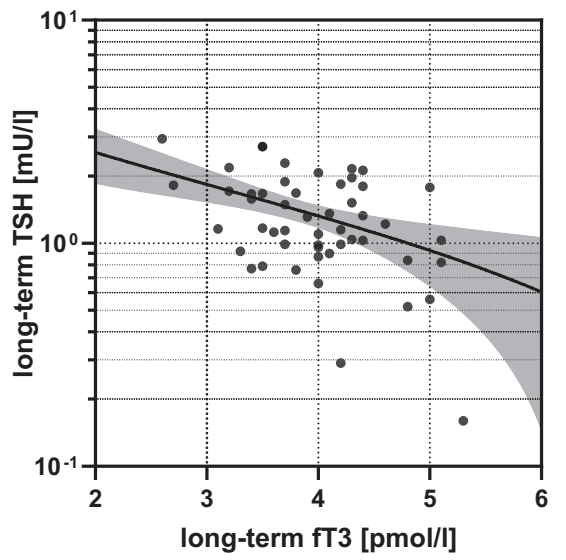

Fig. 2 Log-linear regression analysis between TSH and thyroid hormones at long-time follow-up. a fT3 and TSH-levels, b fT4 and TSHlevels. fT3 free triiodothyronine, fT4 free thyroxine, TSH thyroid stimulating hormone. 
a

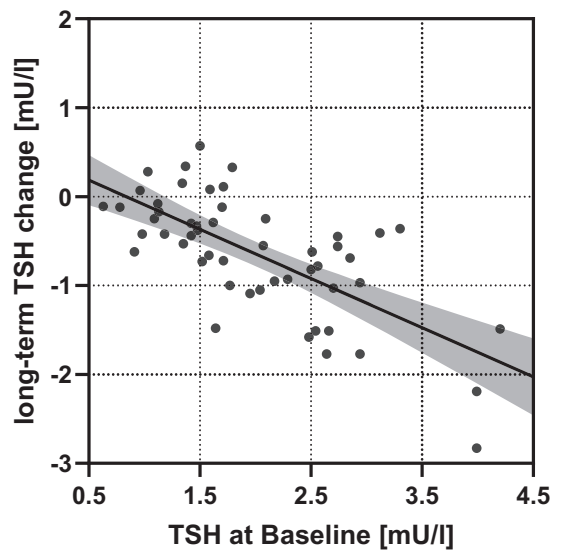

b

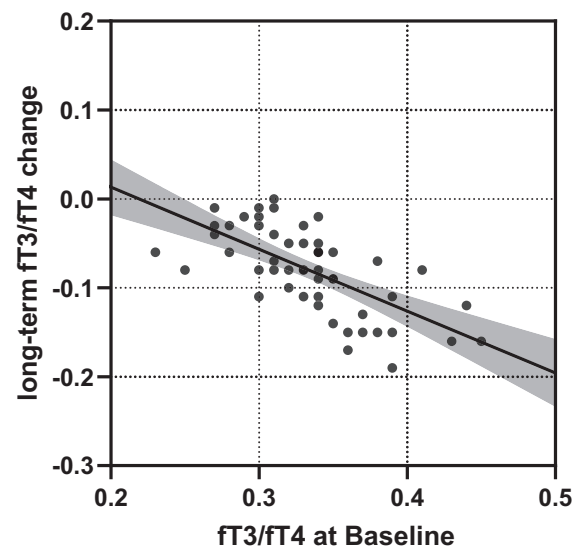

Fig. 3 Linear regression analysis for change in TSH and for change in fT3/fT4-ratio at long-time follow-up. a Linear regression analysis between TSH-change at long-term follow-up and TSH at baseline and $\mathbf{b}$ fT3/fT4-change at long-term follow-up and fT3/fT4 at baseline. TSH thyroid stimulating hormone, fT3 free triiodothyronine, fT4 free thyroxine.

TSH was greater in patients with high-normal TSH values at baseline, suggesting that patients with greater baseline dysregulation will have greater decrease of TSH [13]. Since there is evidence for the association of high-normal TSH with negative cardiovascular and metabolic effects, "normalization" of TSH-levels promoted by BS is particularly important in this at-risk population $[29,30]$.

In our cohort, serum TSH-levels and thyroid hormones fT3 and fT4 presented a strong negative log-linear relation only at 1-year and long-term follow-up. Negative log-linear correlation between serum TSH-levels and thyroid hormone levels has been well described previously in healthy individuals $[22,31,32]$ and reflect the important role of triiodothyronine and thyroxine in the pituitary feedback [33]. Therefore, our data indicates normalization of pituitary feedback and recovery of the pituitary-thyroid-axis 8 years after weight loss surgery. It remains unclear, whether this normalization at long-term was achieved due to weight loss after BS or behavioral and nutritional adjustments during the follow-up period. The impact of nutritional status on the pituitary-thyroidaxis has already been demonstrated before [34-36].

Proposed mechanisms include hypothalamic effects of leptin, which correlates with the percentage of body fat mass and energy balance $[13,35,37]$. TSH-variability in 800 patients with obesity was mainly explained by leptin independently of BMI, suggesting that TSH might be associated with energy balance rather than obesity [37]. Patients with high-normal TSH displayed higher leptin-levels compared to those with normal TSH-values. Following BS, circulating levels of leptin decrease related to weight loss and food restriction leading to reduced stimulation of TRH- and TSH-secretion referred to as the "hypothalamic-pituitary-adiposeaxis" by some authors [37, 38]. Suppression of serum ghrelin following RYGB and SG may have an additive effect on the reduction in TSH-levels [10]. Surprisingly, the course of TSH and fT3/fT4-ratio did not follow the U-shaped curve of weight loss. Furthermore, weight regain at long-term showed no association with the changes in TSH-levels, suggesting thyroid function not to mirror the course of weight loss in the long-term. Thyroid axis function has been previously discussed to interact with food intake and energy expenditure on a more central level and therefore regulating energy balance affected by both, peripheral and central tissue [39]. We can only speculate that food restriction and negative energy balance are key to the favorable long-term effect of BS despite gradual weight regain. Long-term studies that could further elucidate the underlying mechanisms are needed for a thorough understanding of these complex interactions.
Comparing SG and RYGB, there were no significant differences between TSH-levels at long-time in our cohort. This may be explained by the fact that EWL did not significantly differ in patients undergoing RYGB compared to SG 8 years post BS. Moreover, TSH at baseline, which must be considered as the main driver for change in TSH-levels, was not significantly different.

\section{CONCLUSION}

In conclusion, results of the present study reveal a decline in $\mathrm{TSH}-$ levels in euthyroid patients with obesity up to 8 years after BS irrespective of type of surgery and despite gradual weight regain.

Our study has a few limitations. First, our study is a retrospective cohort study, so that internal and external validity may be impaired. Repeat measurements of TSH-levels at baseline were not performed. T3 and thyroid antibodies were not included in the assessment. Vitamin and mineral supplementation, glucocorticoid and psychotropic medication, that may have an influence on thyroid function, were not recorded. Analysis of A1C did not include measurement of hemoglobin.

\section{REFERENCES}

1. World Health Organisation. Obesity and overweight. 2021. https://www.who.int/ en/news-room/fact-sheets/detail/obesity-and-overweight/.

2. Chang S-H, Stoll CRT, Song J, Varela JE, Eagon CJ, Colditz GA. The effectiveness and risks of bariatric surgery. JAMA Surg. 2014;149:275.

3. Michalaki MA, Vagenakis AG, Leonardou AS, Argentou MN, Habeos IG, Makri MG, et al. Thyroid function in humans with morbid obesity. Thyroid. 2006;16:73-8.

4. Biondi B. Thyroid and obesity: an intriguing relationship. J Clin Endocrinol Metab. 2010;95:3614-7.

5. Štěpánek LL, Horáková D, Štěpánek LL, Janout V, Janoutová J, Bouchalová K, et al. Free triiodothyronine/free thyroxine (FT3/FT4) ratio is strongly associated with insulin resistance in euthyroid and hypothyroid adults: a cross-sectional study. Endokrynol Pol. 2020. https://doi.org/10.5603/EP.a2020.0066.

6. Muscogiuri G, Sorice GP, Mezza T, Prioletta A, Lassandro AP, Pirronti T, et al. Highnormal tsh values in obesity: is it insulin resistance or adipose tissue's guilt? Obesity. 2013;21:101-6.

7. Xu R, Huang F, Zhang S, Lv Y, Liu Q. Thyroid function, body mass index, and metabolic risk markers in euthyroid adults: a cohort study. BMC Endocr Disord. 2019;19:58.

8. Janssen IMC, Homan J, Schijns W, Betzel B, Aarts EO, Berends FJ, et al. Subclinical hypothyroidism and its relation to obesity in patients before and after Roux-en-Y gastric bypass. Surg Obes Relat Dis. 2015;11:1257-63.

9. Juiz-Valiña P, Outeiriño-Blanco E, Pértega S, Varela-Rodríguez BM, García-Brao MJ, Mena $E$, et al. Effect of weight loss after bariatric surgery on thyroid-stimulating hormone levels in euthyroid patients with morbid obesity. Nutrients. 2019;11. https://doi.org/10.3390/nu11051121. 
10. Abu-Ghanem Y, Inbar R, Tyomkin V, Kent I, Berkovich L, Ghinea R, et al. Effect of sleeve gastrectomy on thyroid hormone levels. Obes Surg. 2015;25:452-6.

11. Liu F, Di J, Yu H, Han J, Bao Y, Jia W. Effect of Roux-en-Y gastric bypass on thyroid function in euthyroid patients with obesity and type 2 diabetes. Surg Obes Relat Dis. 2017;13:1701-7.

12. Alagna S, Cossu ML, Masala A, Atzeni MM, Ruggiu M, Satta FM, et al. Evaluation of serum leptin levels and thyroid function in morbidly obese patients treated with bariatric surgery. Eat Weight Disord. 2003;8:95-9.

13. Neves JS, Castro Oliveira S, Souteiro P, Pedro J, Magalhães D, Guerreiro V, et al. Effect of weight loss after bariatric surgery on thyroid-stimulating hormone levels in patients with morbid obesity and normal thyroid function. Obes Surg. 2018;28:97-103.

14. MacCuish A, Razvi S, Syed AA. Effect of weight loss after gastric bypass surgery on thyroid function in euthyroid people with morbid obesity. Clin Obes. 2012;2:25-8.

15. Guan B, Chen YY, Yang J, Yang W, Wang C. Effect of bariatric surgery on thyroid function in obese patients: a systematic review and meta-analysis. Obes Surg. 2017;27:3292-305.

16. Dall'Asta C, Paganelli M, Morabito A, Vedani P, Barbieri M, Paolisso G, et al. Weight loss through gastric banding: effects on TSH and thyroid hormones in obese subjects with normal thyroid function. Obesity. 2010;18:854-7.

17. Gniuli D, Leccesi L, Guidone C, laconelli A, Chiellini C, Manto A, et al. Thyroid function and insulin sensitivity before and after bilio-pancreatic diversion. Obes Surg. 2010;20:61-8.

18. Chirurgische Arbeitsgemeinschaft für Adipositastherapie (CA-ADIP), Deutsche Adipositas-Gesellschaft (DAG), Deutsche Gesellschaft für Psychosomatische Medizin und Psychotherapie, Deutsche Gesellschaft für Ernährungsmedizin. S3Leitlinie: Chirurgie der Adip. 2010.

19. Cadena-Obando D, Ramírez-Rentería C, Ferreira-Hermosillo A, Albarrán-Sanchez A, Sosa-Eroza $E$, Molina-Ayala $M$, et al. Are there really any predictive factors for a successful weight loss after bariatric surgery? BMC Endocr Disord. 2020;20:20.

20. Magro DO, Geloneze B, Delfini R, Pareja BC, Callejas F, Pareja JC. Long-term weight regain after gastric bypass: a 5 -year prospective study. Obes Surg. 2008;18:648-51.

21. Wartofsky L, Dickey RA. The evidence for a narrower thyrotropin reference range is compelling. J Clin Endocrinol Metab. 2005;90:5483-8.

22. Baloch Z, Carayon P, Conte-Devolx B, Demers L, Feldt-Rasmussen U, Henry J, et al. Laboratory support for the diagnosis and monitoring of thyroid disease. Thyroid. 2003;13:3.

23. Faul F, Erdfelder E, Lang A-G, Buchner A. G*Power 3: a flexible statistical power analysis program for the social, behavioral, and biomedical sciences. Behav Res Methods. 2007;39:175-91.

24. Chikunguwo SM, Wolfe LG, Dodson P, Meador JG, Baugh N, Clore JN, et al. Analysis of factors associated with durable remission of diabetes after Roux-en- $Y$ gastric bypass. Surg Obes Relat Dis. 2010;6:254-9.

25. Moulin De Moraes CM, Mancini MC, De Melo ME, Figueiredo DA, Villares SMF, Rascovski A, et al. Prevalence of subclinical hypothyroidism in a morbidly obese population and improvement after weight loss induced by Roux-en-Y gastric bypass. Obes Surg. 2005;15:1287-91.

26. Knudsen N, Laurberg P, Rasmussen LB, Bülow I, Perrild H, Ovesen L, et al. Small differences in thyroid function may be important for body mass index and the occurrence of obesity in the population. J Clin Endocrinol Metab. 2005;90:4019-24.

27. Nyrnes A, Jorde R, Sundsfjord J. Serum TSH is positively associated with BMI. Int J Obes. 2006:30:100-5

28. Kuś A, Marouli E, Del Greco MF, Chaker L, Bednarczuk T, Peeters RP, et al. Variation in normal range thyroid function affects serum cholesterol levels, blood pressure, and type 2 diabetes risk: a Mendelian randomization study. Thyroid. 2020. https:// doi.org/10.1089/thy.2020.0393.

29. Cercato C, Fonseca FA. Cardiovascular risk and obesity. Diabetol Metab Syndr. 2019;11. https://doi.org/10.1186/s13098-019-0468-0.

30. Taylor PN, Razvi S, Pearce SH, Dayan CM. A review of the clinical consequences of variation in thyroid function within the reference range. J Clin Endocrinol Metab. 2013;98:3562-71.

31. Spencer CA, LoPresti JS, Patel A, Guttler RB, Eigen A, Shen D, et al. Applications of a new chemiluminometric thyrotropin assay to subnormal measurement. J Clin Endocrinol Metab. 1990;70:453-60.

32. Jonklaas J, Kahric-Janicic N, Soldin OP, Soldin SJ. Correlations of free thyroid hormones measured by tandem mass spectrometry and immunoassay with thyroid-stimulating hormone across 4 patient populations. Clin Chem. 2009;55:1380-8.

33. Larsen PR. Thyroid-pituitary interaction: feedback regulation of thyrotropin secretion by thyroid hormones. N Engl J Med. 1982;306:23-32.

34. Burman KD, Smallridge RC, Osburne R, Dimond RC, Whorton NE, Kesler P, et al. Nature of suppressed TSH secretion during undernutrition: effect of fasting and refeeding on TSH responses to prolonged TRH infusions. Metabolism. 1980;29:46-52

35. Légrádi G, Emerson CH, Ahima RS, Flier JS, Lechan RM. Leptin prevents fastinginduced suppression of prothyrotropin-releasing hormone messenger ribonucleic acid in neurons of the hypothalamic paraventricular nucleus. Endocrinology. 1997;138:2569-76

36. Boelen A, Wiersinga WM, Fliers E. Fasting-induced changes in the hypothalamuspituitary-thyroid axis. Thyroid. 2008;18:123-9.

37. Bétry C, Challan-Belval MA, Bernard A, Charrié A, Drai J, Laville M, et al. Increased TSH in obesity: evidence for a BMI-independent association with leptin. Diabetes Metab. 2015;41:248-51.

38. Schäffler A, Binart N, Schölmerich J, Büchler C. Hypothesis paper: brain talks with fat-evidence for a hypothalamic-pituitary-adipose axis? Neuropeptides. 2005;39:363-7

39. López M, Alvarez CV, Nogueiras R, Diéguez C. Energy balance regulation by thyroid hormones at central level. Trends Mol Med. 2013;19:418-27.

\section{AUTHOR CONTRIBUTIONS}

$A L, F S$, and JA designed the study. $A L, M W$, and $J A$ collected the data. $A L$ and $F S$ performed the statistical analysis and drafted the manuscript. MW, OM, $\mathrm{PB}, \mathrm{TBH}$, and JA critically reviewed the manuscript. All authors read and approved the final manuscript.

\section{FUNDING}

Open Access funding enabled and organized by Projekt DEAL.

\section{COMPETING INTERESTS}

The authors declare no competing interests.

\section{ADDITIONAL INFORMATION}

Correspondence and requests for materials should be addressed to Anne Lautenbach.

Reprints and permission information is available at http://www.nature.com/ reprints

Publisher's note Springer Nature remains neutral with regard to jurisdictional claims in published maps and institutional affiliations.

Open Access This article is licensed under a Creative Commons Attribution 4.0 International License, which permits use, sharing, adaptation, distribution and reproduction in any medium or format, as long as you give appropriate credit to the original author(s) and the source, provide a link to the Creative Commons license, and indicate if changes were made. The images or other third party material in this article are included in the article's Creative Commons license, unless indicated otherwise in a credit line to the material. If material is not included in the article's Creative Commons license and your intended use is not permitted by statutory regulation or exceeds the permitted use, you will need to obtain permission directly from the copyright holder. To view a copy of this license, visit http://creativecommons. org/licenses/by/4.0/

(c) The Author(s) 2022 